\title{
Patient interest in recording family histories of cancer via the Internet
}

Christian Simon, $P h D^{1}$, Louise Acheson, $M D^{2,3}$, Christopher Burant, $P h D^{4}$, Nancy Gerson, $B S^{1}$, Sarah Schramm, $M A^{1}$, Susan Lewis, MSSA ${ }^{5}$, and Georgia Wiesner, $M D^{6,7,8,9}$

\begin{abstract}
Purpose: This study investigated the interest of mammogram patients in using electronic tools for recording their family histories of cancer (FHC). Methods: Semistructured interviews were conducted with 65 patients visiting a breast center at a referral hospital in Cleveland, Ohio. Results: Most $(n=40 ; 62 \%)$ respondents expressed interest in using an electronic tool for recording FHC and associated its use with a range of benefits to themselves, their families, and their health care providers. Women who were not interested $(n=25 ; 38 \%)$ in using an electronic tool for recording FHC were concerned about privacy issues, computer proficiency, and giving up the opportunity to provide family history information directly to the health care provider. Interest in using an electronic tool for recording FHC was not significantly associated with age, race, level of education or income, personal or FHC, or Internet access and frequency of use. Conclusion: Electronic documentation of FHC was seen as largely desirable. However, clinical services to facilitate systematic family history documentation are likely to require more than one avenue for collecting and communicating this information, as not everyone who wants to provide a FHC to a health care provider is comfortable using the Internet to do so. Genet Med 2008:10(12):895-902.
\end{abstract}

Key Words: cancer, family history, Internet

Family histories are an important part of the preventative tool kit for cancers with a genetic link. ${ }^{1-4}$ Detailed family histories of cancer (FHC) can aid in cancer risk assessment, and may help promote cancer screenings and other risk-reducing measures. ${ }^{5-12}$ Recently, electronic tools have been developed specifically for the purposes of facilitating collection and assessment of FHC. ${ }^{5,11,13-19}$ Interest in self-administered tools for recording FHC has been spurred by the advantages that electronic applications offer over more traditional methods of family history taking, including saving time during the clinical encounter, thorough and systematic recording of FHC, the structured data that result, and patient participation and empowerment. ${ }^{13,18,20}$ However, family history taking with computers and the Internet may have perceived drawbacks such as concerns about information security, access to this technology,

\footnotetext{
From the ${ }^{1}$ Department of Bioethics, Case Western Reserve University, Cleveland, Ohio ${ }^{2}$ Department of Family Medicine, Case Western Reserve University, Cleveland, Ohio; ${ }^{3}$ Case Comprehensive Cancer Center, Cleveland, Ohio; ${ }^{4}$ Frances Payne Bolton School of Nursing, Case Western Reserve University, Cleveland, Ohio; ${ }^{5}$ Department of Genetics, Case Western Reserve University, Cleveland, Ohio; and ${ }^{6}$ Department of Genetics, Case Western Reserve University, Cleveland, Ohio; ${ }^{7}$ Case Comprehensive Cancer Center, Cleveland, Ohio; ${ }^{8}$ Center for Human Genetics, Cleveland, Ohio; and ${ }^{9}$ University Hospitals Case Medical Center, Cleveland, Ohio.

Christian Simon, PhD, Department of Internal Medicine, Program in Bioethics and Medical Humanitics, Roy J. and Lucille A. Carver School of Medicine, 500 Hawkins Drive, 1-110 MEB, Iowa City, IA 52242-1190. E-mail: christian-simon@uiowa.edu.

Disclosure: The authors declare no conflict of interest.

Submitted for publication July 24, 2008.

Accepted for publication September 15, 2008.

DOI: 10.1097/GIM.0b013e31818de708
}

usability, and other issues. These issues may inhibit interest and uptake of electronic tools for recording FHC, unless they are anticipated and addressed with the help of empirical data.

This study investigated the interest of mammogram patients in providing their FHC via electronic tools specifically designed for recording FHC. A detailed FHC is often the first step in identifying hereditary breast cancer susceptibility and deleterious BRCA gene mutations. The US Preventive Services Task Force and other professional organizations recommend referring women with family histories suggesting hereditary breast and ovarian cancer for genetic counseling and possible testing. ${ }^{21}$ The importance of early detection and screening for hereditary cancers is paramount. ${ }^{3,22-24}$ Mammogram patients and/or their families are one group who may benefit from the use of electronic tools for recording FHC. ${ }^{25,26}$ The overall goal of the study was to explore mammogram patients' views, attitudes, and concerns surrounding possible use of an electronic tool for recording their FHC. One hypothesis of the study was that patients' experience with computer use and access to the Internet would have an effect on their interest in using an electronic tool for recording their FHC. Findings of the study are intended to inform the design and implementation of a selfadministered electronic tool for recording and assessing FHC called the Genetic Risk Easy Assessment Tool (GREAT) being developed at a nonprofit university-based hospital in Cleveland, Ohio. The GREAT uses a validated questionnaire at a secure Internet site to record and analyze personal and family medical history. A personal report is generated including a family tree, risk assessment for breast, ovarian, and colon cancer, and prevention messages. 


\section{MATERIALS AND METHODS}

The study sample consisted of women seeking mammograms at a university hospital-based breast diagnostic center in Cleveland, Ohio. Semistructured interviews were conducted with a convenience sample of 65 women who visited the breast center for routine mammograms or follow-up purposes.

\section{Instrument}

Interviews were completed using a 49-item interview guide that addressed the following domains of interest: subjects' personal and FHC; reason(s) for seeking a mammogram; attitudes toward the privacy and confidentiality of their FHC in general; prior use of an electronic tool for recording FHC, if any; attitudes toward using an electronic tool for recording FHC and sharing family history information electronically; access to and use of a computer and the Internet; and personal demographics.

Because the study was aimed at exploring how perceptions of the Internet may affect patient interest in recording their FHC, the study instrument probed only patients' perceptions of electronically recording their FHC, and not the element of risk assessment as well. Patients who had never used or been offered the use of an electronic tool for recording FHC were read a paragraph that preempted the questions that followed, which stated:

"I would like you to imagine that your doctor asks you to provide your family history of cancer using a computer that is hooked up to the Internet. The computer has a special online program for recording family histories of cancer."

A literature review was conducted to ascertain if there existed other interview guides or measures designed to explore these domains of interest. None specific to electronic family cancer history recording were found; however, several questions related to technology and Internet health use generally were available and were included in the study questionnaire. ${ }^{27,28}$ The interview questions on privacy and confidentiality of family histories and electronic tools for recording FHC specifically were independently developed and iteratively pilot tested over the course of 2 months. A team of seven investigators and research staff, including a medical anthropologist, geneticist, statistician, and an oncologist and family physician, met periodically to design the questionnaire items, review the results of each of two cycles of pilot testing, and finalize the instrument.

\section{Recruitment}

The study was approved by the Institutional Review Board and the director of the breast center at the institution where the study was conducted. Recruitment took place in the waiting room of the breast center over 7 months between 2007 and 2008. Two study recruiters visited the breast center twice a day (once in the morning and once in the afternoon) on 4 days a week (Monday-Thursday). The recruiters approached patients in the mammogram waiting room and briefly explained the study to them. Patients were asked if they were interested in learning more about the study and were provided with information packets if they were interested. The packets contained a cover letter, two copies of the informed consent document, an interview scheduling sheet, and a self-addressed, stamped envelope. Recruiters asked interested patients for their permission to be contacted via telephone. Interested patients were asked to review and sign a copy of the consent form and mail it back in the self-addressed, stamped envelope. All potential participants were given an opportunity to ask questions about the study before deciding whether or not to participate. Interviews were completed over the telephone except in the case of five participants, who requested a face-to-face interview. The average interview time was 23 minutes and 25 seconds $(\mathrm{SD}=5$ minutes and 44 seconds). Study participants were compensated $\$ 25$ for participating in the study.

\section{Data management and analysis}

Interviews were digitally recorded and stored in .wma files. Interview responses to closed-ended questions were transcribed into an SPSS 13.0 data file and checked for accuracy and completeness. Qualitative, open-ended responses were manually transcribed into Word. A second research assistant validated the transcriptions by checking each one against the original audiotaped version. The quantitative outcome of interest in this study was whether or not individuals would be interested in using a computer-based program to record their FHC. Because the current study was exploratory in nature, the set of predictors covered a wide range of areas that may influence an individual's interest in using a computer to record FHC. The predictors focused on the following areas: demographics (ethnicity, age, education, and income); personal history of cancer; FHC; general Internet access and use; general privacy of family history information; use of electronic tools for recording FHC specifically; and opinions about access of researchers to electronic family histories. The $\chi^{2}$ statistic was run for all categorical and dichotomous outcomes. Likert scales were treated as categorical. $t$ Tests were run for all continuous variables for comparisons based on whether or not a subject was interested in using a computer program to record FHC.

Qualitative data were analyzed using standard iterative coding $^{29}$ of women's responses to the question, "Why are you/are you not interested in using a computer-based program to record your family history of cancer?" Specifically, the responses to this question were first grouped into two broad categories, interested and not interested. The responses were then analyzed by a research assistant and the study PI (CS) for explanations as to why respondents were either interested or not interested in using an electronic tool for recording their FHC. Several categories of explanations emerged from this analysis, which were then presented to the full research team for critical discussion and revision. The final agreed-upon categories and the explanations contained in them were then independently coded for verifiability and formatted as a table (see Table 2). 


\section{RESULTS}

\section{Sample characteristics}

A total of 154 women were approached; 65 (42\%) consented and were interviewed. Among the nonparticipants, women either explicitly stated that they were not interested in participating in the study $(n=41 ; 46 \%)$, were lost to telephone follow-up ( $n=38 ; 43 \%)$, or cited personal or family circumstances as a reason for not participating in the study $(n=10 ; 11 \%)$.

Demographic data on those who participated in the study are presented in Table 1. Twenty-six (40\%) of these interviewed women identified themselves as African American and the remainder as white. The average age of women was 57 years (range, 36-89 years of age). Women gave various reasons for visiting the breast center, including to have an annual mammogram $(n=38 ; 59 \%)$, a follow-up visit for additional testing $(n=23 ; 35 \%)$, or some other reason, such as a physician referral for a specific problem $(n=4 ; 6 \%)$. Most $(n=39 ; 60 \%)$ women reported no personal history of cancer of any kind. The rest reported having had either breast cancer $(n=20 ; 31 \%)$, leukemia $(n=2 ; 3 \%)$, cervical cancer $(n=2 ; 3 \%)$, or thyroid cancer $(n=2 ; 3 \%)$. The majority $(n=56 ; 86 \%)$ of women in the sample reported having at least one family member with cancer.

Eighty percent $(n=52)$ of the women said that they were regular Internet users and most $(n=46 ; 71 \%)$ had Internet access at home. However, African American women were less likely than white women to have a computer at home $\left(\chi^{2}=\right.$ 13.48; $P<0.01)$ and to have experience using the Internet $\left(\chi^{2}=\right.$ 13.48; $P<0.01)$.

\section{Views on two different avenues of providing FHC}

Almost all ( $n=63 ; 97 \%)$ of the study participants reported having at some point provided information about their FHC to a health care provider. Women felt either "very comfortable" $(n=58 ; 89 \%)$ or "mostly comfortable" $(n=5 ; 8 \%)$ with the prospect of providing this information directly to their health care provider. Only two (3\%) women felt "uncomfortable." Furthermore, almost all $(n=61 ; 94 \%)$ thought that their family members would also be comfortable with them sharing the FHC with a health care provider. Most considered their FHC to be "not so private" ( $n=28 ; 43 \%)$ or "not private at all" ( $n=$ $28 ; 43 \%)$, whereas eight $(12 \%)$ said that it was either "very private" or "absolutely private."

Although none of the women in the sample reported ever having used or been offered the use of an electronic tool for recording their FHC, the majority $(n=40 ; 62 \%)$ expressed an interest in using such a tool. When asked why they were interested, women felt that a computerized tool would probably be more efficient, save time, and/or speed dissemination of cancer history data (see Table 2). Another commonly given explanation was that the cancer histories could be of benefit to the patient's family and to other women with cancer, the patient's community or culture, health care providers or researchers, and future generations of women.
Table 1

Sample characteristics $(N=65)$

\begin{tabular}{lc}
\hline & $N(\%)$ \\
\hline Personal history of cancer & $26(40)$ \\
Yes & \\
Family history of cancer (FHC) & $56(86)$ \\
Yes & $37(57)$ \\
$\quad$ First degree relative & $19(29)$ \\
Distant relative & \\
Demographics & $56.68 \pm 11.22(36-89)$ \\
Age (yrs), mean \pm SD (range) & \\
Ethnicity & $26(40)$ \\
African American & $39(60)$ \\
Caucasian &
\end{tabular}

Education

Less than high school
High school diploma/GED
Some College
College undergraduate degree
Graduate school or more

Income

$\$ 20,000$ or less

$\$ 20,000$ to less than $\$ 50,000$

$11(17)$

$\$ 50,000$ or more

Internet access and use

Do you use the Internet?

Yes

How often do you go online?

Daily

Several times a week

$11(17)$

Once a week or less

Never

Do you have a computer at home? Yes

Is there Internet access in your home?

Yes

$46(71)$

Not all women in the sample were interested in using an electronic tool for recording their FHC. Some women $(n=25$; $38 \%$ ) expressed reservations about providing their FHC to their health care provider via an online computer program. Notably, this group included both of the women who said they were "uncomfortable" with providing their FHC directly to their health care provider $(n=2 ; 3 \%)$. Women who said they were not interested in using an online computer program to record their FHC provided a range of explanations 
Table 2

Reasons patients gave for being/not being interested in using an online computer program to record their family history of cancer

\begin{tabular}{|c|c|c|}
\hline Thematic categories & $\begin{array}{l}\text { No. } \\
\text { responses }\end{array}$ & Example \\
\hline \multicolumn{3}{|l|}{ Reasons why patients were interested } \\
\hline \multirow[t]{2}{*}{ Advantages of using a computer/going online } & \multirow[t]{2}{*}{12} & "I just think it might be a more efficient way of documenting it." \\
\hline & & "It's a quick way of getting it to a number of doctors and researchers." \\
\hline \multirow[t]{2}{*}{ To help other people } & \multirow[t]{2}{*}{12} & "... the only purpose in having that information is to help other people." \\
\hline & & "I want to help others in my culture." \\
\hline \multirow[t]{2}{*}{ To inform and help family } & \multirow[t]{2}{*}{9} & "It would just be a good record to pass on to my family, my children, my grandchildren." \\
\hline & & $\begin{array}{l}\text { "It's important to ... come up with genetic reasons why my family would get cancer or } \\
\text { have cancer in the future, in my children's future, just so that I can provide } \\
\text { information that would help." }\end{array}$ \\
\hline To help cancer research & 9 & $\begin{array}{l}\text { "The cancer rates are so astronomical I think it's definitely got to be something.... } \\
\text { environmental and I think the more they look into it, they could maybe pinpoint a } \\
\text { reason ... so I think the research is definitely necessary." }\end{array}$ \\
\hline Other reasons & 5 & "It's something new, something different." \\
\hline \multicolumn{3}{|l|}{ Reasons why patients were not interested } \\
\hline Lack of computer use/experience & 7 & “No, cause I'm not that swift with it ... I don't do that well with computers, no." \\
\hline Confidentiality issues & 7 & "It's just not something I want on the Internet .... The Internet is too open ...." \\
\hline Not applicable to my patient/family & 5 & $\begin{array}{l}\text { "Cause I know what the family history is, I don't think I need to record it ... I mean, it's } \\
\text { just a couple people." }\end{array}$ \\
\hline \multirow[t]{2}{*}{ Other reasons } & \multirow[t]{2}{*}{8} & "Because I just don't have the time." \\
\hline & & "I'm just not comfortable with it." \\
\hline
\end{tabular}

for their response, including that they lacked the necessary computer skills, were concerned about online security, or did not need a cancer risk assessment, among other explanations (see Table 2).

\section{Differences between those interested and not interested in using an electronic tool for recording their FHC}

We statistically compared those women who were and were not interested in using an electronic tool for recording their FHC in an effort to identify additional possible explanations for their interest or lack thereof. Three factors were significantly associated with interest in using this kind of tool (see Table 3). First, those interested $(n=21 ; 53 \%)$ in recording their FHC using an electronic tool were more willing than those not interested $(n=3 ; 12 \%)$ to forego a face-to-face meeting with their health care provider to record their FHC $\left(\chi^{2}=\right.$ 10.84; $P<0.01)$. Second, no women interested in using an electronic tool for recording their FHC also reported feeling uncomfortable with the idea of sharing their FHC with their health care provider, whereas at least some $(n=2 ; 8 \%)$ women not interested in using this kind of tool reported being uncomfortable with sharing their FHC with their health care provider $\left(\chi^{2}=6.4 ; P=0.04\right)$. Another factor associated with women's interest in using an electronic tool for recording their FHC was the location in which patients would utilize this service. Those interested in recording their $\mathrm{FHC}$ using an electronic tool were more likely to say they would use their home computer $(n=$
22; 55\%), when compared with those who were not interested $\left(n=7 ; 28 \% ; \chi^{2}=3.79 ; P=0.05\right)$.

Several independent variables were also marginally associated with women's interest in using an electronic tool for recording their FHC (see Table 3). Specifically, when compared with the women who were interested in using an electronic tool, a higher proportion of those who were not interested wanted their health care provider to explain how their computerized FHC would be kept private and confidential $\left(\chi^{2}=\right.$ $3.3 ; P=0.07)$, that the health care provider ask their permission before allowing researchers to access their FHC $\left(\chi^{2}=3.3\right.$; $P=0.07$ ), and that the health care provider explain who will be able to access their history and what they will use it for $\left(\chi^{2}=\right.$ 2.6; $P=0.11$.

A number of likely predictors were not, in fact, significantly associated with whether or not a woman was interested in using an electronic tool for recording their FHC, including her age, race, level of education, or income, and whether or not she personally had had cancer or had a FHC. There were also no significant differences based on whether patients had a first degree relative with cancer, a distant relative with cancer, or no FHC. Self-reports of computer and Internet access and use were also not significantly different between those interested in using an electronic tool for recording FHC and those who were not. Both groups tended to have a computer with high-speed Internet access at home and go online more than once a week (see Table 3). African American women, who were less likely to 
Table 3

Interest in using an electronic tool for recording FHC in association with other patient perceptions and views

Would you be interested in using a computer program to record your family history of cancer?

$\begin{array}{cc}\text { Yes }(n=40) & \text { No. }(n=25) \\ n(\%) & n(\%)\end{array}$

$n(\%)$ $n(\%)$

Significant findings

Privacy issues

How comfortable would you be providing your health care provider with information about your FHC?

Very comfortable

Mostly comfortable

Uncomfortable

Very uncomfortable

Using an electronic tool for recording FHC

How would you feel about using a computer program to record your FHC instead of meeting with your health care provider and recording it face to face with him/her?

In favor

Not in favor

How comfortable would you be recording, storing and sharing your family's history of cancer on a computer and the Internet?

Very/mostly comfortable

Uncomfortable/very uncomfortable

If you were to use a computer with Internet access to record your FHC, where would you prefer to do this?

On a home computer

At the health care provider's office

Nonsignificant findings

Privacy issues

How important is it to you that your health care provider explains to you how your computerized FHC will be kept private and confidential?

Very/somewhat important

Not very/not at all important

How important is to you that your health care provider asks your permission before giving researchers access to your family history of cancer?

Very/somewhat important

Not very/not at all important

How important is it to you that your health care provider explains who will have access to your FHC and what they will use it for?

Very/somewhat important

Not very/not at all Important

Internet use and access

Do you use the Internet?

$\begin{array}{cc}35(88) & 23(92) \\ 5(12) & 0(0) \\ 0(0) & 2(8) \\ 0(0) & 0(0)\end{array}$

$(0)$ 
Table 3

Continued

\begin{tabular}{|c|c|c|c|}
\hline & \multicolumn{3}{|c|}{$\begin{array}{l}\text { Would you be interested in using a computer } \\
\text { program to record your family history of } \\
\text { cancer? }\end{array}$} \\
\hline & $\begin{array}{c}\text { Yes }(n=40) \\
n(\%)\end{array}$ & $\begin{array}{c}\text { No. }(n=25) \\
n(\%)\end{array}$ & $P$ \\
\hline How often do you go online? & & & 0.98 \\
\hline Daily & $17(43)$ & $10(40)$ & \\
\hline Several times a week & $7(17)$ & $4(16)$ & \\
\hline Once a week or less & $8(20)$ & $6(24)$ & \\
\hline Never & $8(20)$ & $5(20)$ & \\
\hline Do you have a computer at home? & & & 0.52 \\
\hline Yes & $33(83)$ & $19(76)$ & \\
\hline No & $7(17)$ & $6(24)$ & \\
\hline Is there Internet access in your home? & & & 0.86 \\
\hline Yes & $29(88)$ & $17(89)$ & \\
\hline No & $4(12)$ & $2(11)$ & \\
\hline
\end{tabular}

have an online computer at home, were just as likely as white women to express an interest in using an electronic tool for recording their FHC.

\section{DISCUSSION}

FHC are potentially important to the early detection and screening of a number of genetically linked cancers, including breast cancer. ${ }^{1}$ With $80 \%$ of American Internet users, or some 113 million adults, having searched the Internet for health information, ${ }^{30}$ electronic tools for creating and documenting FHC have the potential to reach and help large numbers of people. However, the successful adoption of these tools is also dependent on a range of factors, including how interested people are in using them and the specific benefits or drawbacks they associate with using them.

This exploratory study showed that almost all mammogram patients who were interviewed were comfortable with the idea of providing their FHC directly to their health care provider. Few had privacy or confidentiality concerns with this avenue. Furthermore, when asked whether they would be interested in providing their FHC to their health care provider via an online computer program, $62 \%$ were interested. This interest was not confined to women of high income, educational attainment, or one race. Some women associated benefits with the provision of FHC to their health care provider regardless of whether or not they provided these histories directly or via an online computer program. Some mentioned specific benefits in using a web-based tool to save and communicate this information. Many viewed communicating their family history as a vehicle for helping others, including their own family, other families like theirs, or for contributing to medical research. These data suggest that there is interest across a spectrum of mammogram patients in recording FHC via the Internet.

The study also suggests that not all mammogram patients share this interest and that some may have reservations about using an electronic tool for recording their FHC owing to concerns about information security, lack of experience with computers, and giving up the opportunity to provide family history information directly to the health care provider. Interestingly, these women were on average no different in their general attitudes toward technology and their level of access to and use of the Internet than those women who were interested in providing their FHC using an online computer program. This finding suggests that, in this population, computer literacy and Internet access per se may not be the most important predictors of whether mammogram patients are or are not interested in using an online computer program to record their FHC. As a result, a challenge of research on the clinical utility of electronic tools for recording FHC will be to characterize the contexts in which they are deployed, with regard to what users (and nonusers) understand about security and privacy of online information, and the means whereby the computer tool is integrated into clinical care or research.

A number of limitations of this study need to be noted. First, the questions about interest in electronic tools for recording FHC were hypothetical, and the proportion who would actually use such a tool in different settings and formats may be lower, as observed, for example, in a multicenter study of the CDCs online familial risk assessment tool. ${ }^{31}$ In currently ongoing research in this breast center, we will assess the opinions of actual users and nonusers of the aforementioned GREAT. Jones et al..$^{26}$ and Dominguez et al. ${ }^{25}$ reported a large series of women who used a personal computer in the mammography 
facility for family history risk assessment, showing the feasibility of such an approach.

Second, this was an exploratory study with limited statistical power to compare the characteristics of groups with different opinions. Nonetheless, the sample size and its diversity in terms of age, socioeconomic status, and race were adequate for capturing a full range of qualitative explanations as to why mammogram patients may or may not be interested in using an electronic tool for recording their FHC. Third, one should generalize cautiously from this study given that it was limited to female mammogram patients, a group with reason to be interested in cancer risk and recommended screening guidelines. Clearly, future investigations need to determine whether similar variations in attitude are evident among those with different demographic characteristics, and especially among people for whom prevention and early detection is paramount. Although African Americans and whites in this group were equally likely to be interested in using an electronic tool for recording their FHC, broadening this work to include other ethnic groups and nationalities, and observing their actual uptake of an electronic tool for recording FHC is important.

\section{CONCLUSIONS}

Among our sample of women recruited at a referring hospital's breast center, online documentation of FHC was seen as desirable by the majority. Many were interested in the possible benefits of using a computer or the Internet to record this information. Most did not envision problems related to privacy when giving family history information to health care providers via the Internet. A substantial minority, however, are not interested in using such a tool. The most common reasons were lack of computer experience and concerns about information security on the Internet. These barriers, however, are likely to be eradicated over time, as peoples' computer expertise continues to improve. Patients may need to be educated on using a computer and on the security of information on the Internet. A longitudinal study would be useful in determining if these issues can be, or already have been, overcome.

There is a need for more in-depth investigation of the relationship between the reasons behind people's concerns about computer and Internet use (not focused on access alone) and their uptake of electronic family history recording for cancer risk assessment. This study suggests that clinical services to facilitate systematic family history assessment are likely to require more than one avenue for collecting and communicating this information, ${ }^{32}$ as not everyone who wants to provide family history to a health care provider is comfortable using a computer or the Internet to do so.

\section{ACKNOWLEDGMENTS}

This project was supported by a New Voices Faculty Seed Grant (SG-2) from the Center for Genetic Research Ethics and Law (CGREAL) at Case Western Reserve University, made possible by NIH Grant P50-HG-03390 from the National Human Genome Research Institute.
We thank the patients who participated in this project. We are also grateful for input from Michelle McGowan, Audrey Lynn, and Eric Juengst, among others.

\section{References}

1. Chew H. Genetic evaluation of cancer: the importance of family history. Texas Med 2001;97:40-45.

2. Eberl M, Sunga A, Farrell C, Mahoney M. Patients with a family history of cancer: identification and management. J Am Board Fam Pract 2005;18:211-217.

3. Negri E, Braga C, LaVecchia C, Franceschi S, Parazzini F. Family history of cancer and risk of breast cancer. Int J Cancer 1997;72:735-738.

4. Ramsey S, Yoon P, Moonesinghe R, Khoury M. Population-based study of the prevalence of family history of cancer: implications for cancer and screening and prevention. Genet Med 2006;8:571-575.

5. Acheson L, Zyzanski S, Stange K, Deptowicz A, Wiesner G. Validation of a selfadministered, computerized tool for collecting and displaying the family history of cancer. J Clin Oncol 2006;24:5395-5402.

6. Dorman S. Web-based health calculators. J School Health 2001;71:500-501.

7. Emmons K, Wong M, Puleo E, Weinstein N, Fletcher R, Colditz G. Tailored computer-based cancer risk communication: correcting colorectal cancer risk perception. J Health Commun 2004;9:127-141.

8. Gomez E. Internet offers interactive screening programs and risk assessments. ONS News 2001;16:5.

9. Johnson C, Ensor J, Amos C, et al. An informatics tool to assess individualized colon cancer risk. AMIA Annu Symp Proc 2005:998.

10. Nelson H, Huffman L, Fu R, Harris E. Genetic risk assessment and BRCA mutation testing for breast and ovarian cancer susceptibility: systematic evidence review for the U.S. Preventive Services Task Force. Ann Intern Med 2005;143:362-379.

11. Skinner C, Rawl S, Moser B, et al. Impact of the Cancer Risk Intake System on patient-clinician discussions of tamoxifen, genetic counseling, and colonoscopy. J Gen Int Med 2005;20:360-365.

12. Westman J, Hampel H, Bradley T. Efficacy of a touchscreen computer based family cancer history questionnaire and subsequent cancer risk assessment. J Med Genet 2000;37:354-360.

13. Kelly K, Sweet K. In search of a familial cancer risk assessment tool. Clin Genet 2007;71:76-83.

14. O'Neill S. Communicating familial risk-mygenerations. Paper presented at Family History Tools to Improve the Public's Health. Atlanta, GA, November 17, 2005.

15. Emery J, Walton R, Murphy M, et al. Computer support for interpreting family histories of breast and ovarian cancer in primary care: comparative study with simulated cases. BMJ 2000;321:28-32.

16. Emery J, Morris H, Goodchild R, et al. The GRAIDS trial: a cluster randomized controlled trial of computer decision support for the management of familial cancer risk in primary care. Br J Cancer 2007;97:486-493.

17. Hughes K. Breast/ovarian cancer risk assessment tool (AVON). Paper presented at Family History Tools to Improve the Public's Health. Atlanta, GA, November 17, 2005.

18. Qureshi N, Wilson B, Santaguida P, et al. Collection and use of cancer family history in primary care. Rockville, MD: Agency for Healthcare Research and Quality, 2007.

19. Yoon P, Scheuner M, Jorgensen C, Khoury M. Healthware (TM). A family history screening tool for the prevention of common chronic diseases. Chronic Disease Prevention. In press.

20. Rich E, Burke W, Heaton C, et al. Reconsidering the family history in primary care. J Gen Int Med 2004;19:273-280.

21. US Preventive Services Task Force. Genetic risk assessment and BRCA mutation testing for breast and ovarian cancer susceptibility: recommendation and statement. Ann Intern Med 2005;143:355-361.

22. Dapic V, Carvalho M, Monteiro A. Breast cancer susceptibility and the DNA damage response. Cancer Control 2005;12:127-136.

23. Shah M, Zhu K, Palmer R, Jatoi I, Shriver C, Wu H. Breast, colorectal, and skin cancer screening practices and family history of cancer in U.S. women. J Womens Health 2007;16:526-534.

24. Sifri R, Gangadharappa S, Acheson L. Identifying and testing for hereditary susceptibility to common cancers. CA Cancer J Clin 2004;54:309-326.

25. Dominguez F, Jones J, Zabicki K, et al. Prevalence of hereditary breast/ovarian carcinoma risk in patients with a personal history of breast or ovarian carcinoma in a mammography population. Cancer 2005;104:1849-1853.

26. Jones J, Hughes K, Kopans D, et al. Evaluation of hereditary risk in a mammography population. Clin Breast Cancer 2005;6:38-44.

27. Simon C, Schramm S. Cancer and the computerized family: towards a clinical ethics 


\section{Simon et al.}

of "indirect" Internet use. Medicine, Health Care and Philosophy. Available at: http://dx.doi.org/10.1007/s11019-008-9127-1. Accessed February 19, 2008.

28. Bishop L, Holmes B, Kelley C. National Consumer Health Privacy Survey 2005. Oakland, CA: California HealthCare Foundation, 2005.

29. Simon C, Schramm S. Cancer and the computerized family: towards a clinical ethics of "indirect" Internet use. Med Health Care Philos 2008;11:337-341.

30. Fox S. Online Health Search 2006. Pew Internet \& American life project. Available at:
http://www.pewinternet.org/pdfs/PIP_Online_Health_2006.pdf. Accessed October 29, 2006.

31. O’Neill S, Rubinstein W, Wang C. Familial risk for common diseases in primary care: the Family Healthware (TM) Impact Trial. In press.

32. Cohn W, Jones S, Miesfeldt S. “Are you at risk for hereditary breast cancer?” Development of a personal risk assessment tool for hereditary breast and ovarian cancer. J Genetic Couns 2008;17:64-78. 